\title{
A NEW ANALYTICAL EXPRESSION FOR THE T.C.R. OF THIN MONOCRYSTALLINE METAL FILMS
}

\author{
C. R. TELLIER \\ Universite de Nancy 1, Laboratoiré d'Electronique \& I.G.B.M., \\ Case Officielle No 140 - 54037 Nancy Cedex, France
}

(Received March 8, 1978; in final form June 1, 1978)

\begin{abstract}
The analysis of electrical conductivity of continuous thin monocrystalline metal film has been treated by assuming that the scattering from other sources than grain-boundaries can be described by an effective relaxation time. This relaxation time method is applied to the temperature coefficient of resistivity and leads to an analytical approximate equation in terms of the grain-boundary reflection coefficient $r$ and the reduced thickness $k$.

Comparison of the results with those deduced from the exact equation (derived from the Mayadas and Shatzkes theory) shows that they deviate by less than $5 \%$ in large $k-, p-$, and $r$-ranges.
\end{abstract}

\section{INTRODUCTION}

In the past few years experimental results on the size and grain-boundary effects in electrical conduction of monocrystalline thin metallic films ${ }^{1-6}$ have been analyzed by a theory proposed by Mayadas and Shatzkes. ${ }^{1,8}$ In the Mayadas Shatzkes (M-S) model three types of electron scattering mechanisms are taken into account i.e., a background, a grainboundary and an external surfaces scattering. However this model leads to a rather complicated expression of the thin monocrystalline film conductivity. Hence, we have previously proposed ${ }^{7}$ an approximate formulation based on the relaxation time method. By assuming that the scattering from sources other than grain-boundaries can be described by an effective relaxation time and by imposing the Boltzmann equation boundary conditions due to the grain-boundaries, ${ }^{8}$ we have obtained an analytical expression of the thin monocrystalline film conductivity $\sigma_{F m}$ in terms of the reduced thickness $k=a / \ell_{0}$ and the physical parameter $\alpha$, i.e.

$$
\sigma_{F m} / \sigma_{0}=[1-A(k)] \cdot f[B(k)]
$$

where $1-A(k)$ is the Fuchs function, i.e.

$$
A(k)=\frac{3}{2 k}(1-p) \int_{1}^{\infty} \cdot\left(\frac{1}{t^{3}}-\frac{1}{t^{5}}\right) \frac{1-e^{-k t}}{1-p e^{-k t}} d t
$$

and

$$
B(k)=\alpha[1-A(k)]
$$

$$
f(B)=1-\frac{3}{2} B+3 B^{2}-3 B^{3} \ln \left(1+\frac{1}{B}\right)
$$

where $p$ is the specularity parameter. ${ }^{9}$ The $\alpha$ parameter is related to the bulk mean free path $\ell_{0}$, to the average grain diameter $a_{g}$ (identified with the film thickness $a$ in monocrystalline films) and to the "grain-boundary reflection coefficient $r$ " by Eqs. (5),

$$
\alpha=\frac{\ell_{0}}{a_{g}} \frac{r}{1-r}
$$

i.e. for monocrystalline films

$$
\alpha=\frac{\ell_{0}}{a} \frac{r}{1-r}=\frac{1}{k} \frac{r}{1-r}
$$

\section{A GENERAL EXPRESSION FOR THE T.C.R. OF A THIN MONOCRYSTALLINE FILM}

The thin film temperature coefficient of resistivity (t.c.r.) $\beta_{F}$ is defined by ${ }^{10}$

$$
\beta_{F}=\frac{1}{\rho_{F}} \frac{d \rho_{F}}{d T}
$$

where $T$ is the temperature.

The t.c.r. $\beta_{0}$ of the bulk material is

$$
\beta_{0}=\frac{1}{\rho_{0}} \frac{d \rho_{0}}{d T}
$$


Assuming that the rigid band model of metals is valid and that the number of conduction electrons per unit volume is temperature independent in the experimental range, the bulk t.c.r. $\beta_{0}$ can be rewritten in the usual form:

$$
\beta_{0}=-\frac{1}{\ell_{0}} \frac{d \ell_{0}}{d T}
$$

With the assumptions that the thermal coefficient of grain-boundary reflection coefficient $r$ and linear expansion of the film are negligible compared to $1 / \ell_{0} d \ell_{0} / d T$ and that there is no mismatch in thermal expansion coefficient between the film and the substrate we can write Eq. (8).

$$
\begin{aligned}
& d k=-\frac{k}{\ell_{0}} d l_{0}=k \cdot \beta_{0} \cdot d T \\
& d \alpha=\frac{d \alpha}{d k} \cdot d k=-\alpha \cdot \beta_{0} \cdot d T
\end{aligned}
$$

Hence, logarithmic differentiation of Eq. (1) gives

$$
\frac{d \sigma_{F m}}{\sigma_{F m}}-\frac{d \sigma_{0}}{\sigma_{0}}=\frac{d\{1-A(k)\}}{1-A(k)}+\frac{d f[B(k)]}{f[B(k)]}
$$

with

$$
\frac{d\{1-A(k)\}}{1-A(k)}=-\frac{k}{1-A(k)} \cdot \frac{d\{1-A(k)\}}{d k} \cdot \frac{d \ell_{0}}{\ell_{0}}
$$

and

$$
\frac{d f[B(k)]}{f[B(k)]}=\frac{1}{f[B(k)]} \cdot \frac{d f[B(k)]}{d B(k)} \cdot d B(k)
$$

in which

$$
\begin{gathered}
d B(k)=\left\{-\frac{d[1-A(k)]}{d k} \cdot k \cdot \alpha+[1-A(k)] \cdot \alpha\right\} \\
\frac{d l_{0}}{\ell_{0}}
\end{gathered}
$$

Using the relations

$$
\begin{aligned}
& -E(k)=\frac{k}{1-A(k)} \frac{d\{1-A(k)\}}{d k} \\
& g[B(k)]=B(k) \cdot \frac{d f[B(k)]}{d B(k)}
\end{aligned}
$$

i.e.

$$
g(B)=-\frac{3}{2} B+6 B^{2}+\frac{3 B^{3}}{1+B}-9 B^{3} \ln \left(1+\frac{1}{B}\right)
$$

Eq. (10) can be rewritten simply as

$$
\begin{aligned}
\frac{d \sigma_{F m}}{\sigma_{F m}}-\frac{d \sigma_{0}}{\sigma_{0}} & =\frac{d \ell_{0}}{\ell_{0}}\left\{E(k)\left[1+\frac{g[B(k)]}{f[B(k)]}\right]\right. \\
& \left.+\frac{g[B(k)]}{f[B(k)]}\right\}
\end{aligned}
$$

giving

$$
\beta_{F m / \beta_{0}}=\left[1+\frac{g[B(k)]}{f[B(k)]}\right] \cdot[1+E(k)]
$$

Contrary to the general Mayadas-Shatzkes expression for the thin monocrystalline film t.c.r. previously derived $^{11,12}$ and given in Eq. (17), Eq. (16) can be regarded as an analytical expression. This is discussed in Section 3.

$$
\beta_{F m} / \beta_{0}=1+\frac{g(\alpha)-A^{*}+B^{*}+C^{*}}{f(\alpha)-A^{*}}
$$

with

$$
\begin{aligned}
A^{*}= & \frac{6}{\pi k}(1-p) \int_{0}^{\pi / 2} d \phi \int_{1}^{\infty} d t \frac{\cos ^{2} \phi}{H^{2}(t, \phi)}\left(\frac{1}{t^{3}}-\frac{1}{t^{5}}\right) \\
& \frac{1-\exp \{-k t H(t, \phi)\}}{1-p \exp \{-k t H(t, \phi)\}}
\end{aligned}
$$$$
B^{*}=\frac{6}{\pi}(1-p)^{2} \int_{0}^{\pi / 2} d \phi \int_{1}^{\infty} d t \frac{\cos ^{2} \phi}{H^{2}(t, \phi)}\left(\frac{1}{t^{2}}-\frac{1}{t^{4}}\right)
$$

$$
\begin{gathered}
\frac{\exp \{-k t H(t, \phi)\}}{[1-p \exp \{-k t H(t, \phi)\}]^{2}} \\
C^{*}=\frac{12 \alpha}{\pi k}(1-p) \int_{0}^{\pi / 2} d \phi \int_{1}^{\infty} \frac{\cos \phi}{H^{3}(t, \phi)}\left(\frac{1}{t^{2}}-\frac{1}{t^{4}}\right) .
\end{gathered}
$$

$$
\frac{1}{\left(t^{2}-1\right)^{1 / 2}} \times \frac{1-\exp \{-k t H(t, \phi)\}}{1-p \exp \{-k t H(t, \phi)\}} d t
$$

$$
H(t, \alpha)=1+\frac{\alpha}{\cos \phi \cdot\left(1-\frac{1}{t^{2}}\right)^{1 / 2}}
$$




\section{DISCUSSION}

In the Fuchs-Sondheimer (F-S) theory the thin film conductivity has been evaluated to be

$$
\sigma_{F}^{*}=\sigma_{0}[1-A(k)]
$$

Hence, we can write

$$
\frac{d \sigma_{F}^{*}}{\sigma_{F}^{*}}=\frac{d \sigma_{0}}{\sigma_{0}}+\frac{d\{1-A(k)\}}{1-A(k)}
$$

and from Eq. (11) and (13), it is seen that, in the F-S model the film t.c.r. $\beta_{F}$ is

$$
\beta_{F}^{*} / \beta_{0}=1+E(k)
$$

The function $E(k)$ can be rewritten in the general form

$$
E(k)=\frac{-A(k)+B(k)}{1-A(k)}
$$

with

$$
B(k)=\frac{3}{2}(1-p) \int_{1}^{\infty} d t\left(\frac{1}{t^{2}}-\frac{1}{t^{4}}\right) \frac{e^{-k t}}{\left[1-p e^{-k t}\right]^{2}}
$$

The $1+E(k)$ function has been intensively tabulated by several authors ${ }^{13-16}$ in the case of completely diffuse ${ }^{13,16}$ or partially specular ${ }^{14,15}$ scattering on external surfaces. Also it will be seen that the thin monocrystalline film t.c.r. $\beta_{F m}$ can be easily evaluated from the analytical expression, Eq. (16), without writing a program for a digital computer as recently reported by other authors. ${ }^{11}$

Note that the model presented here satisfies essential qualitative physical requirements, i.e.

1) When the surface scattering is entirely specular (i.e. $p=1$ ) Eq. (16) reduces to Eq. (21).

$$
\beta_{F m} / \beta_{0}=1+\frac{g(\alpha)}{f(\alpha)}
$$

which is the result previously obtained ${ }^{17}$ for $\beta_{g}$, the grain-boundary t.c.r. (without $\mathrm{F}-\mathrm{S}$ effect).

2) When the grain-boundary reflection coefficient $r$ approaches zero (i.e. $\alpha \approx 0$ ) Eq. (16) can be rewritten in the form:

$$
\beta_{F m} / \beta_{0} \approx 1+E(k)
$$

and the t.c.r., $\beta_{F m}$, coincides with the $\mathrm{F}-\mathrm{S}$ t.c.r., $\beta_{F}^{*}$, as expected.

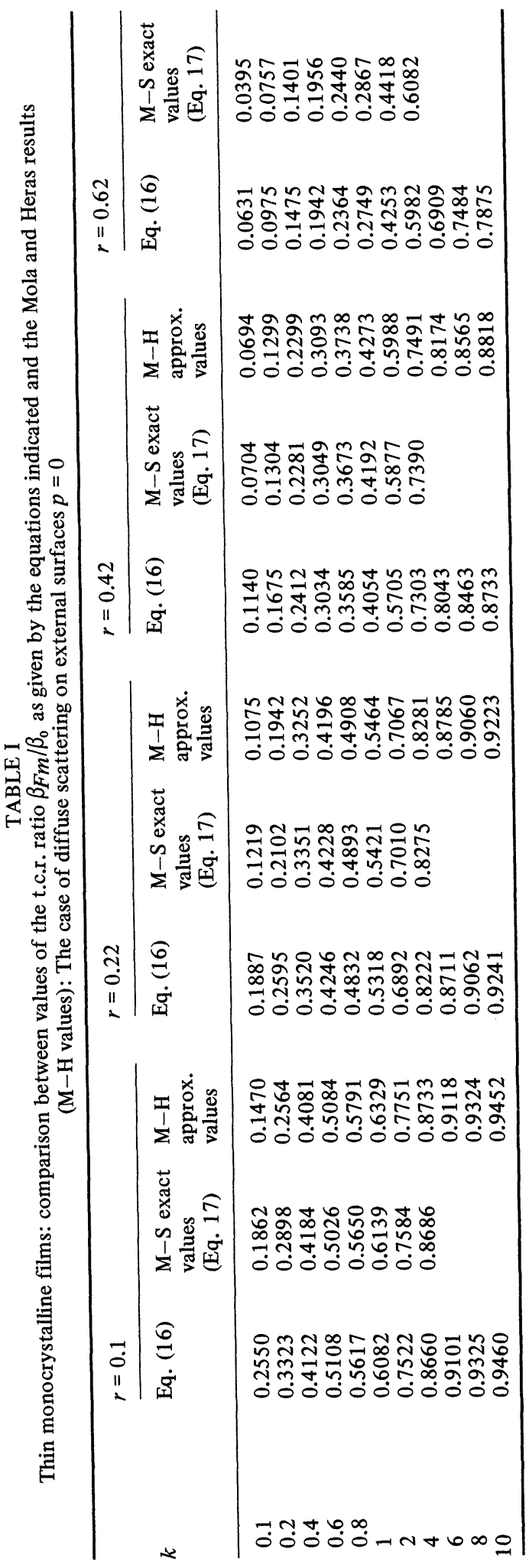


TABLE II

Thin monocrystalline films: comparison between values of the t.c.r. ratio $\beta_{F m} / \beta_{0}$ as given by the equations indicated: The case of partially specular scattering on external surfaces: $p=0.5$

\begin{tabular}{|c|c|c|c|c|c|c|c|c|}
\hline \multirow[b]{2}{*}{$k$} & \multicolumn{2}{|l|}{$r=0.1$} & \multicolumn{2}{|l|}{$r=0.22$} & \multicolumn{2}{|l|}{$r=0.42$} & \multicolumn{2}{|l|}{$r=0.62$} \\
\hline & Eq. 16 & $\begin{array}{l}\mathrm{M}-\mathrm{S} \text { exact } \\
\text { values } \\
\text { (Eq. 17) }\end{array}$ & Eq. 16 & $\begin{array}{l}\text { M-S exact } \\
\text { values } \\
\text { (Eq. 17) }\end{array}$ & Eq. 16 & $\begin{array}{l}\text { M-S exact } \\
\text { values } \\
\text { (Eq. 17) }\end{array}$ & Eq. 16 & $\begin{array}{l}\text { M-S exact } \\
\text { values } \\
\text { (Eq. 17) }\end{array}$ \\
\hline 0.1 & 0.3064 & 0.2603 & 0.2011 & 0.1616 & 0.1072 & 0.0845 & 0.0549 & 0.0432 \\
\hline 0.2 & 0.4170 & 0.3962 & 0.2956 & 0.2733 & 0.1702 & 0.1547 & 0.0918 & 0.0825 \\
\hline 0.4 & 0.5512 & 0.5486 & 0.4249 & 0.4205 & 0.2697 & 0.2651 & 0.1557 & 0.1516 \\
\hline 0.6 & 0.6338 & 0.6363 & 0.5127 & 0.5154 & 0.3470 & 0.3485 & 0.2100 & 0.2104 \\
\hline 0.8 & 0.6907 & 0.6948 & 0.5771 & 0.5826 & 0.4094 & 0.4114 & 0.2583 & 0.2610 \\
\hline 1 & 0.7326 & 0.7369 & 0.6266 & 0.6330 & 0.4608 & 0.4672 & 0.3011 & 0.3052 \\
\hline 2 & 0.8340 & 0.8453 & 0.7662 & 0.7707 & 0.6244 & 0.6311 & 0.4584 & 0.4628 \\
\hline 4 & 0.9155 & 0.9166 & 0.8671 & 0.8693 & 0.7663 & 0.7697 & 0.6245 & 0.6270 \\
\hline 6 & 0.9427 & & 0.9076 & & 0.8302 & & 0.7104 & \\
\hline 8 & 0.9567 & & 0.9292 & & 0.8664 & & 0.7644 & \\
\hline 10 & 0.9652 & & 0.9425 & & 0.8899 & & 0.8011 & \\
\hline
\end{tabular}

Numerical values of $\beta_{F m} / \beta_{0}$ for the thin monocrystalline film have been calculated from Eq. (16) for different values of the specularity parameter, $p$, and grain-boundary reflection coefficient, $r$. The results from Eq. (16) are compared with both those of the M-S theory (as tabulated by Mola and Heras ${ }^{11}$ ) and with those (Table I) deduced from the Mola and Heras linearized approximate equation ${ }^{11}$ which can be only applied in the case of entirely diffuse scattering on external surfaces $(p=0)$.

Recently Dobierzewska-Mozrzymas and Warkusz ${ }^{6}$ have proposed an analytical expression for the resistivity of monocrystalline films by assuming that the external size effect is determined by Cottey's function. ${ }^{18}$ In this paper, we have not used this method to derive an analytical relation for the t.c.r. because it is now well established that the Cottey relation constitutes a good approximation of the F-S relation only in the case of nearly specular scattering on external surfaces $(p \geqslant 0.8)$.

It is shown in Tables I and II that for low values of the reduced thickness $k$ and large values of the grainboundary reflection coefficient $r$, the $\beta_{F m} / \beta_{0}$ ratio values decrease with increasing values of the specularity parameter $p$. In this $k$-range the $1-A(k)$ function is generally evaluated with a higher error, hence a relatively high deviation of the $1+g\{B(k)\} /$ $f\{B(k)\}$ function is expected for $k<0.1$. On the other hand, at room temperature, metallic films thinner than $100 \AA$ (i.e. $k$ generally lower than 0.5 ) are discontinuous and electrical conduction in such films involves several complicated processes which cannot be analyzed in terms of $\mathrm{F}-\mathrm{S}$ or $\mathrm{M}-\mathrm{S}$ effect theory.
From examination of Tables I and II it follows that the greater the value of $p$, the lower the deviation from the exact $\mathrm{M}-\mathrm{S}$ values (Eq. 17). For example, for $0.5 \leqslant k \leqslant 10$ and for $0.1 \leqslant r \leqslant 0.42$ we generally obtain a deviation less than $3 \%$ with $p=0$ and less than $1.5 \%$ with $p=0.5$. However for relatively high values of the grain-boundary reflection coefficient $r(r>0.42)$ the percentage deviation from the $\mathrm{M}-\mathrm{S}$ function increases (i.e. $4.5 \%$ for $r=0.62$ in the case of diffuse scattering on external surfaces).

\section{CONCLUSION}

It is now well established ${ }^{19-24}$ that thin metallic films thicker than $100 \AA$ (i.e. $k>0.5$ ) may be regarded as continuous. Furthermore in the $0.5-10$ range of values for $k$, Eq. (16) deviates by only $5 \%$ in the case of diffuse scattering and $2 \%$ in the case of partially specular scattering $(p=0.5)$ and this result is verified in a large $r$ range $(0.1 \leqslant r<0.62)$. As a consequence of the above it appears that the procedure previously proposed ${ }^{7}$ for analyzing the monocrystalline film resistivity is also applicable to the t.c.r. and constitutes a convenient method to obtain a simple expression of the thin monocrystalline film t.c.r. in large $k$ - and $r$ - ranges.

\section{REFERENCES}

1. A. F. Mayadas and M. Shatzkes, Phys. Rev. B, 1 (1970), 1382.

2. E. E. Mola, J. Borrajo and J. M. Heras, Surface Sci., 34 (1973), 561. 
3. A. F. Mayadas, J. F. Janak and A. Gangulee, J. Appl. Phys., 45 (1974), 2780.

4. R. Suri, A. P. Thakoor and K. L. Chopra, J. Appl. Phys., 46 (1975), 2574.

5. A. K. Pal and S. Chaudhuri, J. Mater. Sci., 11 (1976), 872.

6. E. Dobierzewska-Mozrzymas and F. Warkusz, Thin Solid Films, 43 (1977), 267.

7. C. R. Tellier, Electrocomp. Sci. \& Technol. - 5(1978),127

8. A. F. Mayadas, M. Shatzkes and J. F. Janak, Appl. Phys. Lett., 11 (1969), 345.

9. K. Fuchs, Proc. Cambridge Phil. Qoc., 34 (1938), 100.

10. K. L. Chopra, Thin Film Phenomena (McGraw-Hill, 1969).

11. E. E. Mola and J. M. Heras, Thin Solid Films, 18 (1973), 137.

12. C. R. Tellier and A. J. Tosser, Thin Solid Films, 44 (1977), 141.

13. F. Savornin, Compt. Rend. Acad. Sc., 248 (1959), 2458.
14. D. S. Campbell in "The Use of Thin Films in Physical Investigations" (J. C. Anderson, ed.), Academic Press (1966).

15. C. R. Tellier and C. Boutrit, Private communication.

16. J. Borrajo and J. M. Heras, Thin Solid Films, 18 (1973), 267.

17. C. R. Tellier and A. J. Tosser, Thin Solid Films, 43 (1977), 3.

18. A. A. Cottey, Thin Solid Films, $1(1967 / 68), 297$.

19. K. L. Chopra, L. C. Bobb and M. H. Francombe, J. Appl. Phys., 34 (1963), 1699.

20. B. Laville-Saint-Martin, Thin Solid Films, 6 (1970), 359.

21. C. Reale, Czech. J. Phys. B., 21 (1971), 662.

22. J. P. Chauvineau, P. Croce, G. Devant and M. F. Verhaeghe, J. Vac. Sci. \& Technol, 6 (1969), 776.

23. M. Adamov, B. Perovic and T. Nenadovic, Thin Solid Films, 24 (1974), 89.

24. R. Suri, A. P. Thakoor and K. L. Chopra, J. Appl. Phys., 46 (1975), 2574. 

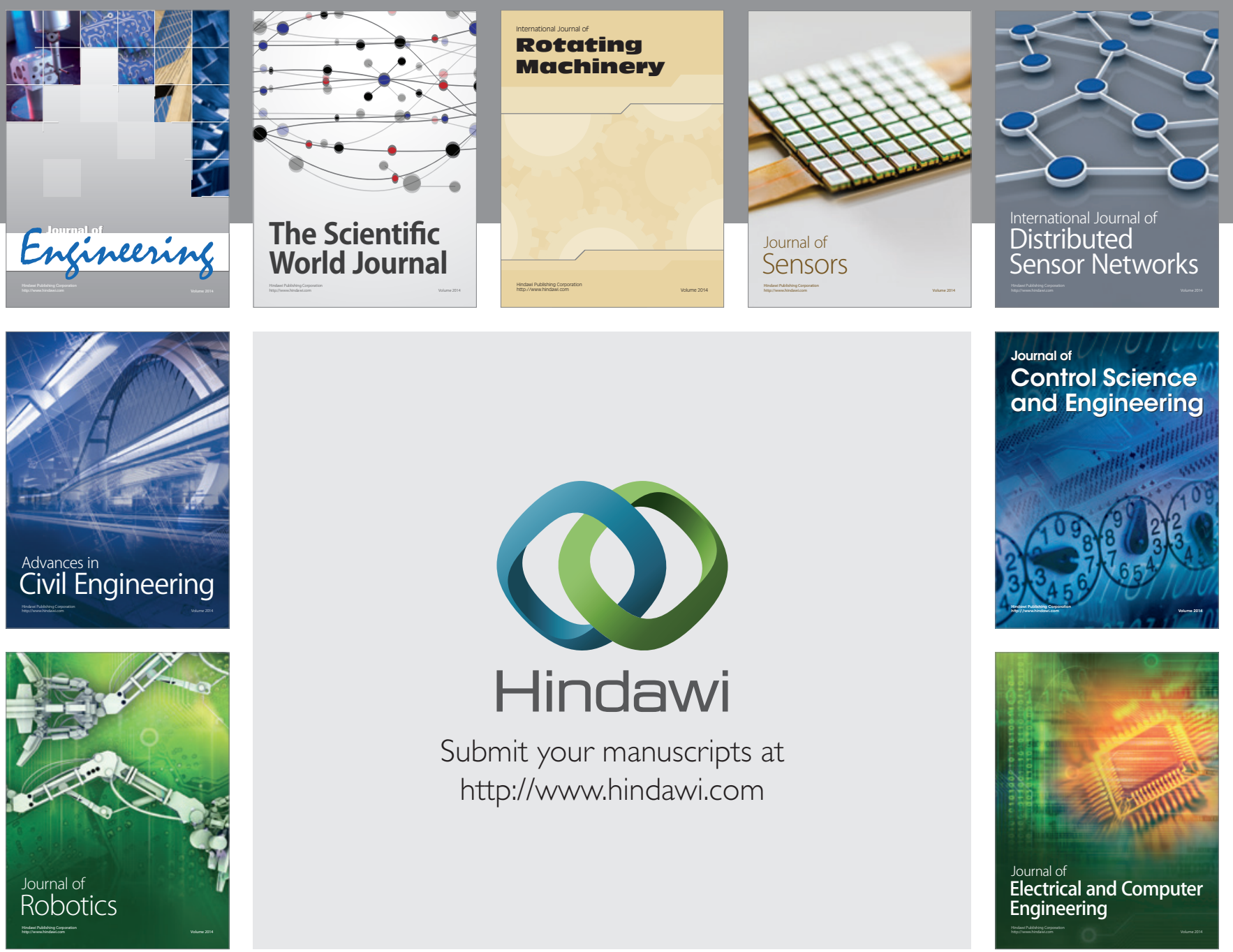

Submit your manuscripts at

http://www.hindawi.com
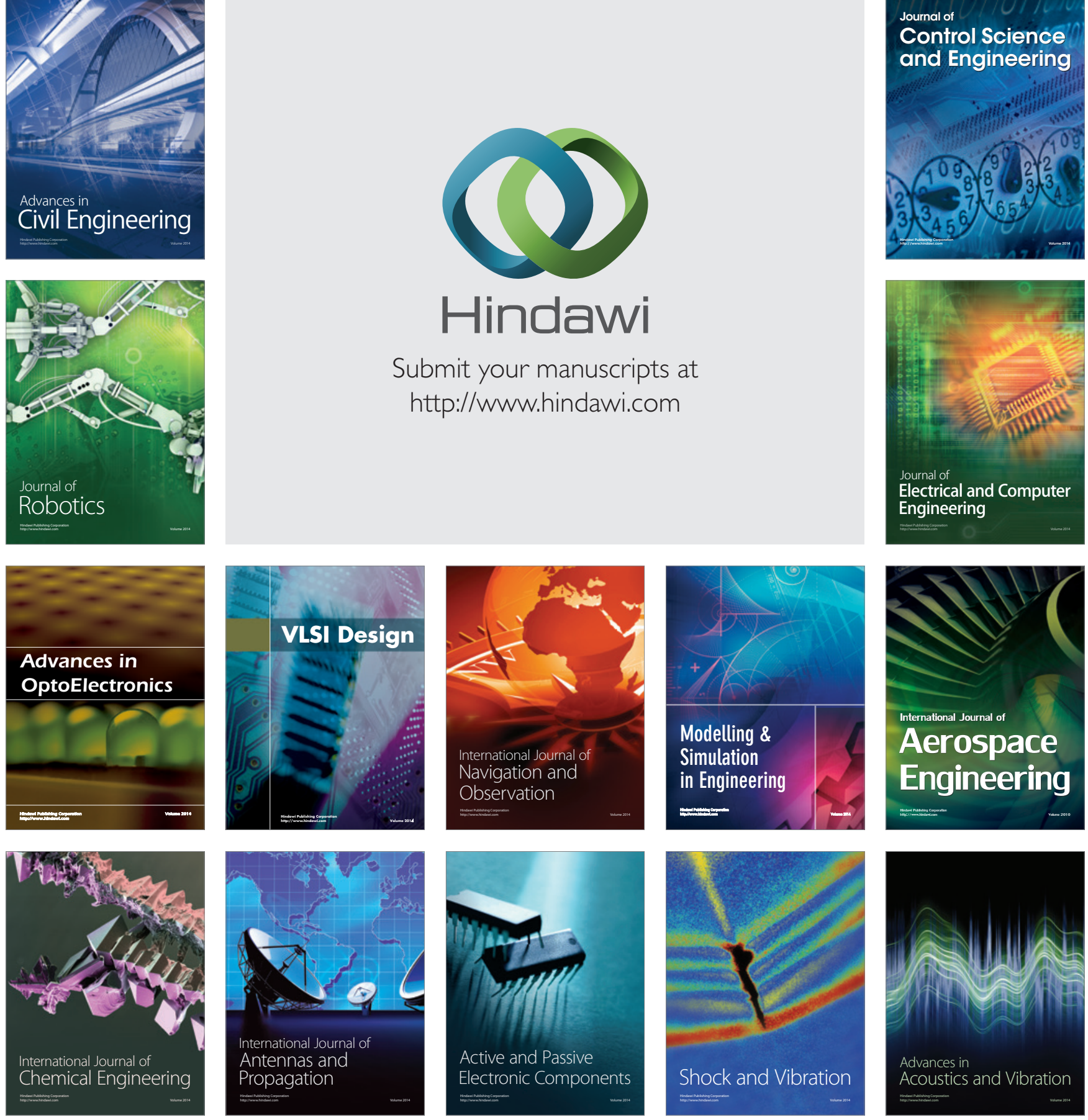